\title{
Acquisition and Chaos-Entropy Analysis of Individuality and Proficiency of Human Operator's Skill Using a Fuzzy Controller
}

\author{
Yoshihiko Kawazoe \\ Saitama Institute of Technology \\ Japan
}

\section{Introduction}

There is an infinite variety of motions ranging from our daily activities to the exceptional movement of an athlete or a musician. Based on his extensive observation of child growth, Gesell (1945) stated some empirical rules. In particular, he noted that the development of motion progresses from a generally integrated state to an individualized state in which individual sections have specialized functions. He also noted that the number of degrees of freedom of the motion increases with development, and that periods of unstabilization and stabilization are repeated to advance development by taking advantage of such fluctuations well. Finally, he observed that chaos plays a very important role in motion. The human process of learning motion can also be studied by focusing on the degrees of freedom. When a person who normally writes with his or her right hand (i.e., their dominant hand) is asked to write with the left hand (i.e., their non dominant hand), the number of degrees of freedom of each joint is initially fixed; but, after training, each joint moves according to a peculiar phase relationship after training (Newell \& Van Emmerik, 1989). This implies that we are rigid when we attempt a new motion, but become more relaxed after getting accustomed to it (Taga, 2002).

Machinery and human beings are absolutely of a different nature at the present stage, but most research work on man-machine systems has dealt with the linear characteristics of human behavior (Kawazoe et al., 2008). As an example, many studies on control systems for stabilizing the inverted pendulum as an inherently unstable system have been presented. These studies focus on the linear characteristics of human behavior. There seem to be few studies and a number of unknowns regarding both the nonlinear characteristics of human behavior in an inherently unstable man-machine system as well as the learning process of human operators with objects difficult to control (Kawazoe et al., 2008, 2009).

In order to stabilize an unstable system such as the inverted pendulum, strict judgment of the situation is required. Accordingly, it can be expected that the human operators exhibit complex behaviors or contingencies, that is, the mixture of regular and random actions intermittently.

The behavior during stabilizing control of an inverted pendulum by a human operator exhibits a random-like or limit-cycle like fluctuation, and the stabilizing control by the human operator is robust against the disturbance. This may be because the limit-cycle-like 
fluctuation produced by the digital PID computer controller, which means lineally unstable, is more robust against the disturbance than the lineally stable fluctuation according to the experiments. The limit cycle was very stable in the sense of nonlinearity, which means it is robust against the disturbance (Kawazoe et al., 1992, 1994, 1999, 2000, 2001a, 2001b).

This chapter investigates the identification of the chaotic characteristics of human operation from the experimental time series data by utilizing fuzzy inference. It shows how to construct rules automatically for a fuzzy controller of each trial of each human operator. It tries to acquire the individual skill of each operator. Human operators in an experiment were trained so that they were skilled to some extent in stabilizing the pendulum by training, and the data of ten trials per person were successively taken for an analysis. The entropy is estimated from the time series data as a measure of the amount of disorder in a system, and the degrees of freedom of the motion are estimated by the dimensions when curves of the largest Lyapunov exponents are saturated against the embedding dimensions for quantifying the proficiency.

\section{Chaos-entropy analysis of human operator's skill during stabilizing control of an inverted pendulum on a cart}

2.1 Trials of stabilizing control of an inverted pendulum on a cart by a human operator Figure 1 shows the experimental setup. An inverted pendulum is mounted on a cart that can move along the line of a sliding rail of limited length. The pendulum is attached to the rail such that the pendulum rotates in one plane. A human operator manipulates the cart directly by hand. Although some time and intensive training are needed in order for a human operator to succeed in stabilizing the pendulum for $60 \mathrm{~s}$, this task becomes less difficult after the first successful instance of stabilization. The human operators in the experiment were trained so that they were skilled to a certain extent in stabilizing the pendulum, and the data obtained in 10 successive trials per person were used for analysis. The angle that the pendulum makes with the vertical axis and the displacement of the cart were measured, and the derivatives and the force that moves the cart can be derived based on these quantities (Kawazoe et al., 2008, 2009).

Figure 2 shows phase plane representations of the chaotic behavior of the inverted pendulum on a cart during stabilizing control by a human operator NK during the first trial after training.

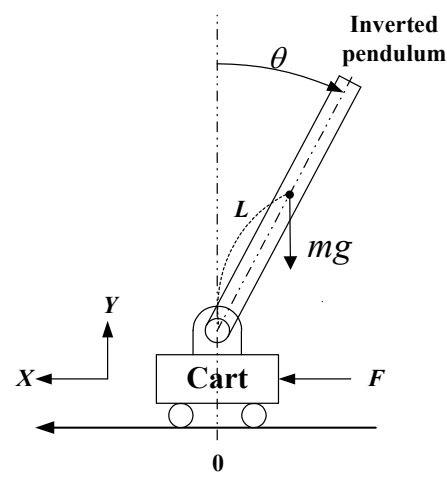

(a)
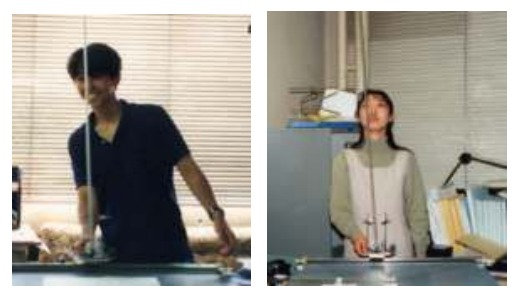

(b)

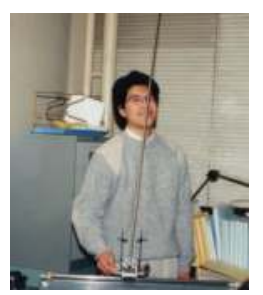

Fig. 1. Stabilizing control of an inverted pendulum. 

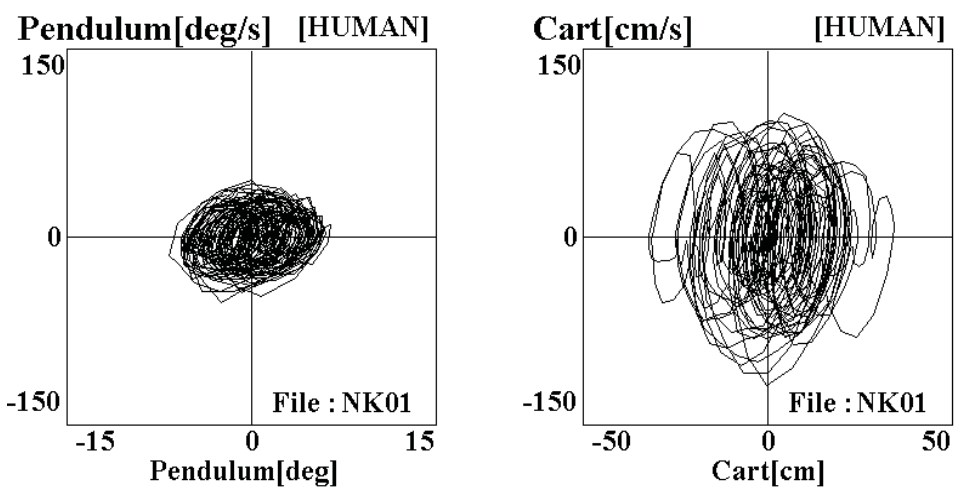

Fig. 2. Behavior of an inverted pendulum in a phase plane.

\subsection{Diagnosis of amount of disorder by entropy analysis}

Consider a hypothetical statistical system in which the outcome of a certain measurement must be located on a unit interval. If a line is subdivided into $N$ subintervals, then we can associate a probability $p_{i}$ with the $i$-th subinterval containing a particular range of possible outcomes. The entropy of the system is then defined as follows:

$$
S=-\sum_{i=1}^{N c} p_{i} \ln p_{i}
$$

This quantity may be interpreted as a measure of the amount of disorder in the system or as the information necessary to specify the state of the system. If the subintervals are equally probable, so that $p_{i}=1 / N$ for all $i$, then the entropy reduces to $S=\ln N$, which can be shown to be the maximum value. Conversely, if the outcome is known to be in a particular subinterval, then $S=0$ is the minimum value. When $S=\ln N$, the amount of further information needed to specify the result of a measurement is at a maximum, and, when $S=$ 0 , no further information is required (Baker et al., 1996; Baierlein, 1997). We applied this formulation to the time series data by establishing $N$ bins or subintervals of unit intervals into which the values of the time series data may fall. We define $S$ as the net entropy calculated using Eq. (1) and $S /\left(\ln \_N\right)$ as the entropy ratio (Kawazoe et al., 2008, 2009). The ratio of entropy to maximum entropy was estimated at the point at which the ratio saturated as the number of partitioned cells increased.

\subsection{Diagnosis of chaotic dynamics by Lyapunov exponent analysis}

The detection of the chaotic dynamics and the quantitative characterization of the chaotic dynamics when the model of the entire system is unknown requires the analysis of time series data. Although methods for dynamic analysis of time series data are currently under development, the following two-step process is commonly used at present: (1) reconstruction of the strange attracter of an unknown dynamic system from the time series, and (2) determination of certain invariant quantities of the system from the reconstructed attracter. It is possible to obtain the dynamics from a single time series without reference to other physical variables (Kawazoe, 1999, 2000). A rigorous mathematical basis of this concept has been presented by Takens (1981) and Mane (1981). 
Since the attracter dimension is unknown for time series data and the required embedding dimension $M$ is unknown, it is important that the reconstruction be embedded in a space of sufficiently large dimension to represent the dynamics completely. Thus, the dimension of the embedding space is increased by increments of one. The attractor is reconstructed, and its largest Lyapunov exponent is calculated. The process is continued until the largest Lyapunov exponent is saturated with respect to the embedding dimensions, and the dimension, i.e., the degrees of freedom of the system behavior, is estimated. The largest Lyapunov exponent can be obtained from time series data using an algorithm presented by Wolf et al. (1985). The Lyapunov exponent can be used to obtain a measure of the sensitivity under the initial conditions. This measure of sensitivity is characteristic of chaotic behavior. If the Lyapunov exponent is positive, nearby trajectories diverge, and so the evolution is sensitive to initial conditions and therefore chaotic.

Consider the time series data $x\left(t_{1}\right), x\left(t_{2}\right), \ldots$ Successive points in the phase space formed from time-delay coordinates can be written in vector form $X_{i}$ as follows:

$$
\begin{aligned}
\mathbf{X}_{1} & =\left(x\left(t_{1}\right), x\left(t_{1}+\tau\right), \cdots, x\left(t_{1}+(m-1) \tau\right)\right) \\
\mathbf{X}_{2} & =\left(x\left(t_{2}\right), x\left(t_{2}+\tau\right), \cdots, x\left(t_{2}+(m-1) \tau\right)\right) \\
\mathbf{X}_{3} & =\left(x\left(t_{3}\right), x\left(t_{3}+\tau\right), \cdots, x\left(t_{3}+(m-1) \tau\right)\right) \\
& \vdots \\
\mathbf{X}_{i} & =\left(x\left(t_{i}\right), x\left(t_{i}+\tau\right), \cdots, x\left(t_{i}+(m-1) \tau\right)\right) \\
& \vdots \\
\mathbf{X}_{N} & =\left(x\left(t_{N}\right), x\left(t_{N}+\tau\right), \cdots, x\left(t_{N}+(m-1) \tau\right)\right)
\end{aligned}
$$

where the symbol $\tau$ denotes the time delay, and the symbol $m$ denotes the embedding dimension.

The choice of an appropriate delay $\tau$ is important to the success of the reconstruction. If $\tau$ is too short, then the coordinates are approximately the same and the reconstruction is useless. If $\tau$ is too large, then the coordinates are so far apart as to be uncorrelated. If the system has some rough periodicity, then a value comparable to but somewhat less than that period is typically chosen. Since there is no simple rule for choosing $\tau$ in all cases, $\tau$ is occasionally adjusted until the results appear to be satisfactory. Time $\tau$ is typically some multiple of the spacing between the time series points (Baker,1996). We chose 7 times the spacing between the time series points, i.e., $7 \times 0.0293 \mathrm{~s}$, as the value of $\tau$ because the calculated largest Lyapunov exponents were not too sensitive to $\tau$ and because the curves of the largest Lyapunov exponents versus embedding dimensions were smooth within a reasonable range, whereas the dominant period of the experimental time series data was 0.5 to $1.0 \mathrm{~s}$. Since the time series is presumed (by hypothesis) to be the result of a deterministic process, each $x_{n+1}$ is the result of a mapping. In other words, we have

$$
x_{n+1}=f\left(x_{n}\right) .
$$

The differentiation of the above equation is approximated as

$$
\frac{d f\left(x_{j}\right)}{d x_{j}}=\frac{d x_{j+1}}{d x_{j}}=\frac{x_{j+1}-x_{j}}{x_{j}-x_{j-1}}=f^{\prime}\left(x_{j}\right) .
$$


Thus, the general expression of the Jacobian matrices and the orthogonal vectors $\mathrm{b}_{i j}(i=1,2, \cdots, m)$ can be obtained (Kawazoe et al., 2008, 2009). The Lyapunov exponents $\lambda_{i}$ for each embedding dimension $i$ are then obtained as (Kawazoe et al., 2008,2009; Baker et al., 1996; Baierlein,1971; Takens, 1981; Mane, 1981; Wolf et al., 1985).

$$
\lambda_{i}=\frac{1}{t_{n}-t_{0}} \sum_{j=1}^{\mathrm{n}-1} \log _{\mathrm{e}} \mathbf{b}_{\mathrm{ij}} \quad(i=1,2,3, \cdots, m) .
$$

The calculated largest Lyapunov exponent converges at the end of the time series data as the embedded dimensions increase. The number of degrees of freedom of motion are estimated by the dimensions when the curves of the largest Lyapunov exponents are saturated with respect to the embedding dimensions.

\section{Generation of a fuzzy controller from time series data during stabilizing control of an inverted pendulum by a human operator}

Fuzzy control has a distinguishing feature in that it can incorporate experts' control rules using linguistic expressions. One of the main problems of fuzzy control is the difficulty in acquiring fuzzy rules and tuning the membership functions. The conventional control theory used to design controllers using models of controlled objects has been established. In addition, a number of studies have examined the design of fuzzy control systems using fuzzy models of controlled objects.

To identify the nonlinear characteristics of the human operator from the experimental time series data, we choose the pendulum angle $\theta_{t}$, angular velocity $\theta^{\prime}{ }_{t}$, and the cart displacement $X_{t}$ and its velocity $X^{\prime}{ }_{t}$ as input variables and the force $F_{t}$ that moves the cart as the output of the fuzzy controller. Furthermore, we choose the combined variables $\theta_{t}+\beta \theta^{\prime}{ }_{t}$ and $X_{t}+\gamma X^{\prime}{ }_{t}$ as inputs so as to reduce the complexity of the control rule table, where $\beta$ and $\gamma$ are combination variables.

The method used to obtain the membership functions and the control rules are described in the following. The values of $\beta$ and $\gamma$ are identified using the identification of membership functions and control rules by a trial and error method after repeating several simulations. In order to partition the data and determine the border of the data with the fuzzy sets for the assumed values of coefficient $\beta$ and $\gamma$, for example, $G_{N B}=10 \%, G_{N S}=25 \%, G_{Z R}=30 \%, G_{P S}=$ $25 \%$, and $G_{P B}=10 \%$ were chosen (Fig.3), and the borders were denoted by $D_{N B}{ }_{N S}, D_{N S} Z R$, $D_{Z R}$ PS, and $D_{P S}$ PB (Fig. 4).

The labels of the membership functions with $\theta+\beta \theta^{\prime}$ and $X+\gamma X^{\prime}$ were determined as follows:

$N B=$ minimum of the data: $D M I N, N S=\left(D_{N B N S}+D_{N S} Z R\right) / 2, Z R=$ mean of the data: $D A V E$, $P S=\left(D_{Z R P S}+D_{P S P B}\right) / 2, P B=$ maximum of the data: $D M A X$.

The labels of the membership function with $F$ are also determined as follows:

$N B=$ minimum of the data: $D M I N, N M B=(N B+N S) / 2, N S=\left(D_{N B N S}+D_{N S} Z R\right) / 2, N M S=$ $N S / 2, Z R=$ average of the data: DAVE, $P M S=P S / 2, P S=\left(D_{Z R P S}+D_{P S} P B\right) / 2, P M B=(P B+$ $P S) / 2, P B=$ maximum of the data: $D M A X$ (Fig. 5).

Suppose that $\theta_{t}+\beta \theta^{\prime}{ }_{t}$ is $G_{N B}, X_{t}+\gamma X^{\prime}{ }_{t}$ is $G_{Z R}$, and $F_{t+1}$ is $G_{N S}$. Then, we count to the cell of label $F=N S$ in the numbered grid to which $\theta+\beta \theta^{\prime}=N B$ and $X+\gamma X^{\prime}=Z R$ are given as inputs. Figure 6 shows the fuzzy output grid numbers for generating a control rule. The 
output is derived using the label frequencies of each grid for each trial and for each human operator and using the following equation (continues to page 334, Eq.(6))

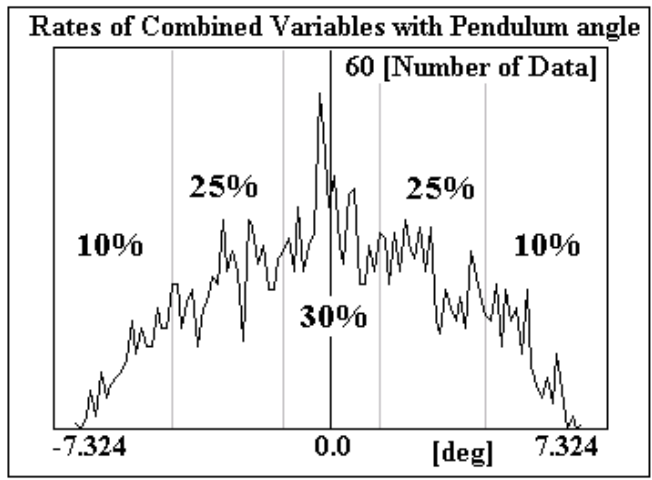

(a) Rates of combined variables with pendulum angle $\theta_{t}+\beta \theta^{\prime}{ }_{t}$

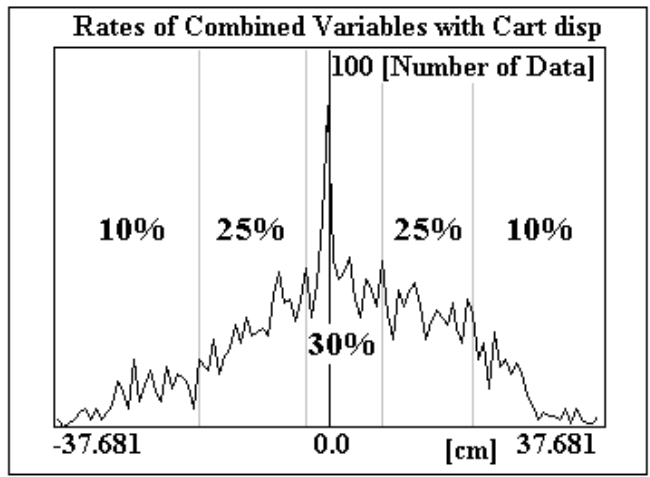

(b) Rates of combined variables with cart displacement $X_{t}+\gamma X^{\prime}{ }_{t}$

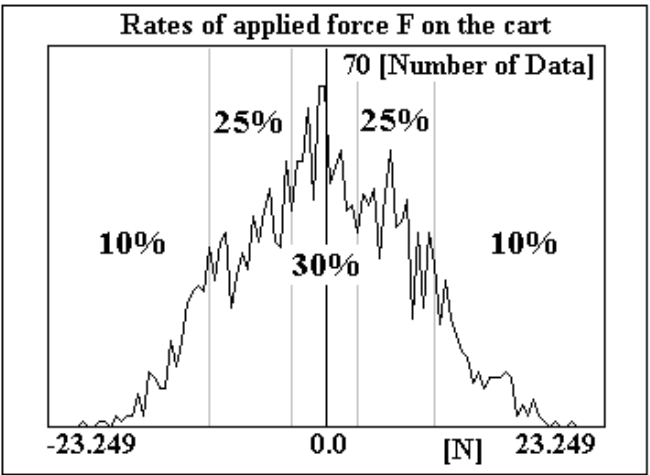

(c) Rates of applied force $F$ on the cart

Fig. 3. Example of rates of inputs and output. 


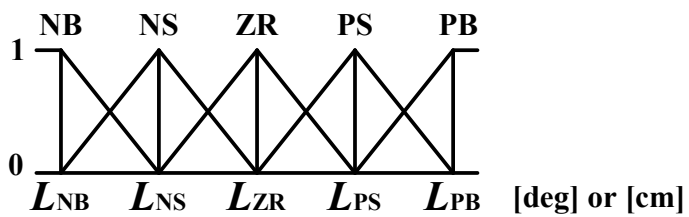

Fig. 4. Membership function for inputs.

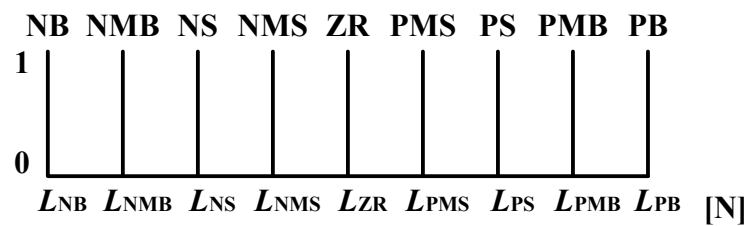

Fig. 5. Membership function (singleton) for output.

\begin{tabular}{|c|c|c|c|c|c|c|}
\hline \multicolumn{2}{|c|}{} & \multicolumn{5}{|c|}{$\theta+\beta \theta^{\prime}$} \\
\cline { 2 - 7 } & N B & N S & Z R & P S & P B \\
\hline & N B & 1 & 2 & 3 & 4 & 5 \\
\hline & N S & 6 & 7 & 8 & 9 & 10 \\
\hline & Z R & 11 & 12 & 13 & 14 & 15 \\
\hline & P S & 16 & 17 & 18 & 19 & 20 \\
\hline & P B & 21 & 22 & 23 & 24 & 25 \\
\hline
\end{tabular}

Fig. 6. Fuzzy output grid number for generating a control rule.

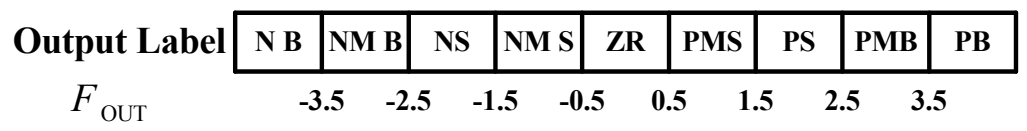

Fig. 7. Conformity of output $F_{\text {out }}$.

\begin{tabular}{|c|c|c|c|c|c|c|}
\hline & \multicolumn{5}{|c|}{$\theta+\beta \theta^{\prime}$} \\
\hline & & $\mathrm{NB}$ & $\mathrm{NS}$ & ZR & PS & PB \\
\hline \multirow{5}{*}{$\begin{array}{l}x \\
7 \\
\pm \\
x\end{array}$} & $\mathrm{NB}$ & PS & PMS & NMS & NMB & ZR \\
\hline & $\mathrm{NS}$ & PMB & PMS & NMS & NMB & ZR \\
\hline & ZR & PB & PS & ZR & $\mathrm{NS}$ & $\mathrm{NB}$ \\
\hline & PS & ZR & PMB & PMS & NMS & NMB \\
\hline & PB & ZR & ZR & PMS & NMS & NS \\
\hline
\end{tabular}

Fig. 8. Rule for control of a pendulum on a cart (first trial NK01 of human operator NK). 


$$
F_{\text {OUT }}=\frac{(-4.4 \cdot \mathrm{NB})+(-2.0 \cdot \mathrm{NS})+(0.0 \cdot \mathrm{ZR})+(2.0 \cdot \mathrm{PS})+(4.4 \cdot \mathrm{PB})}{\mathrm{NB}+\mathrm{NS}+\mathrm{ZR}+\mathrm{PS}+\mathrm{PB}} .
$$

We can determine the output label using Fig.7 and construct the operator's control rule for balancing the inverted pendulum as shown in Fig.8.

Figure 9 shows a block diagram of the stabilizing control simulation of the pendulum on a cart using the constructed fuzzy controller from the time series data of the human operator.

Figure 10 shows the conformity of $\boldsymbol{\theta}+\boldsymbol{\beta} \boldsymbol{\theta}^{\prime}$, and Fig. 11 shows the conformity of $\boldsymbol{X}+\boldsymbol{\gamma} \boldsymbol{X}^{\prime}$, if $\theta+\beta \theta^{\prime}=4.0$ degrees and $\boldsymbol{X}+\boldsymbol{\gamma} \boldsymbol{X}^{\prime}=5.0 \mathrm{~cm}$, as an example. In Fig. 10, the conformity of PS is determined to be 0.70 , and that of PB is determined to be 0.30 . In Fig. 11 the conformity of $\mathrm{ZR}$ is determined to be 0.73 , and that of PS is determined to be 0.27 . Using the fuzzy rule in Fig. 8, the following rules are found:

$$
\begin{gathered}
\text { IF } \theta+\beta \theta^{\prime}=\mathbf{P S} \text { and } X+\gamma X^{\prime}=\mathbf{Z R} \text { THEN } F=\mathbf{N S} \\
\quad \text { else } \\
\text { IF } \theta+\beta \theta^{\prime}=\mathbf{P S} \text { and } X+\gamma X^{\prime}=\mathbf{P S} \text { THEN } F=\mathbf{N M S} \\
\quad \text { else } \\
\text { IF } \theta+\beta \theta^{\prime}=\mathbf{P B} \text { and } X+\gamma X^{\prime}=\mathbf{Z R} \text { THEN } F=\mathbf{N M B} \\
\quad \text { else } \\
\text { IF } \theta+\beta \theta^{\prime}=\mathbf{P B} \text { and } X+\gamma X^{\prime}=\mathbf{P S} \text { THEN } F=\mathbf{N M B}
\end{gathered}
$$

The output values are derived using max-min composition as follows. We use singleton fuzzification as a membership function of output. Figure 12 shows the process called "cutting" by MIN value. Figure 13 shows the process of composition by MAX value. Thus, the membership functions of output referred to as composite fuzzy output are obtained as NMB: 0.30, NS: 0.70, and NMS: 0.27. The output values are calculated using the center of gravity method as follows:

$$
F_{\text {OUT }}=\frac{\sum_{i=1}^{9} X_{i} \cdot Y_{i}}{\sum_{i=1}^{9} Y_{i}},
$$

where the term $X_{i}$ is the $X$ coordinate $(-20.46,-13.37,-6.27,-3.14,-0.02,3.09,6.18,14.72,23.25)$ of the output membership function, $Y_{i}$ is the composite conformity, and $i$ denotes an index. The result of fuzzy inference, $F=-7.28$, is obtained using composite fuzzy output in Fig. 16 . The differential equation of motion of this pendulum-cart system is described as follows:

$$
\begin{gathered}
M \ddot{X}-m L \ddot{\theta} \cos \theta+m L \dot{\theta}^{2} \sin \theta+\mu_{x} \dot{X}=F \\
I \ddot{\theta}-m L \ddot{X} \cos \theta+\mu_{\theta} \dot{\theta}=m g L \sin \theta
\end{gathered}
$$

where $m$ denotes the mass of the pendulum; $M$ denotes the mass of the pendulum, the cart, and a human arm; $L$ is the half-pendulum length; $I$ is the inertial moment of pendulum about the supporting point; $F$ is the force that moves the cart, $\mu_{\theta}$ is the frictional coefficient of the pendulum support point, and $\mu_{X}$ is the frictional coefficient between the cart and the rail. The coefficients $\mu_{\theta}$ and $\mu_{X}$ are derived from the experiment. The sampling time for control is $0.06 \mathrm{~s}$, and the initial pendulum angle is 3.0 degrees. 


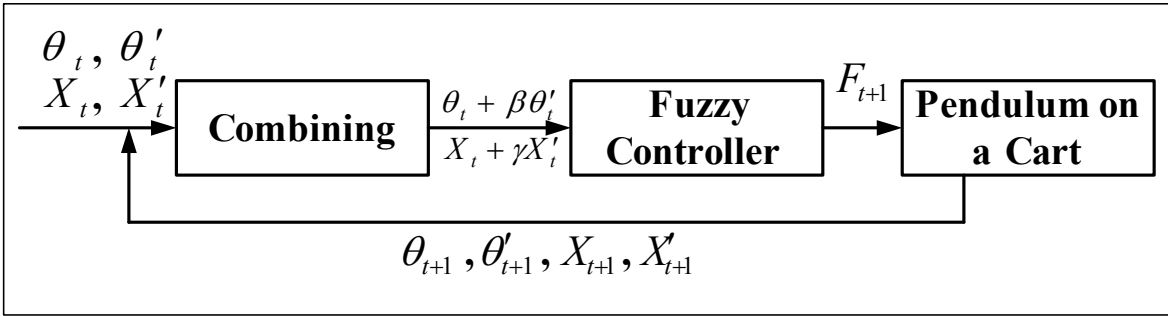

Fig. 9. Stabilizing control simulation of the pendulum using the constructed fuzzy controller from the time series data of a human operator.
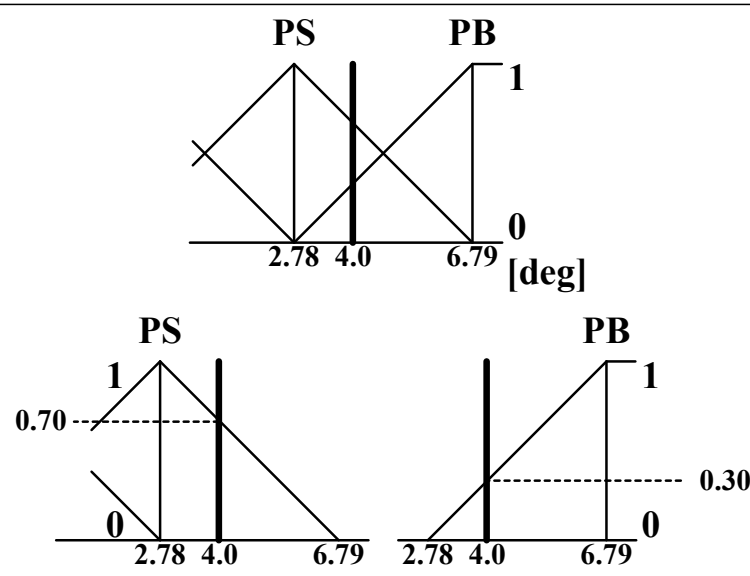

Fig. 10. Conformity of $\boldsymbol{\theta}+\boldsymbol{\beta} \boldsymbol{\theta}^{\prime}$.

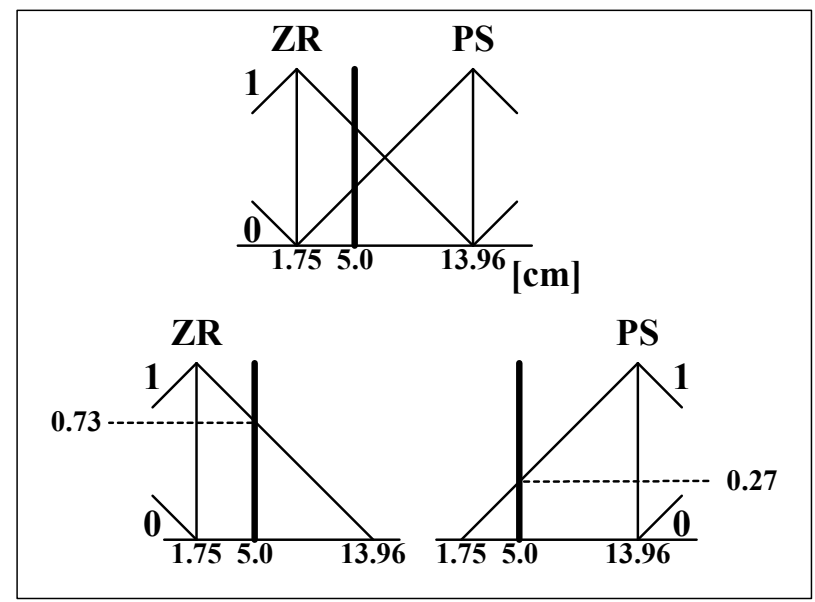

Fig. 11. Conformity of $\boldsymbol{X}+\boldsymbol{\gamma} \boldsymbol{X}^{\prime}$. 


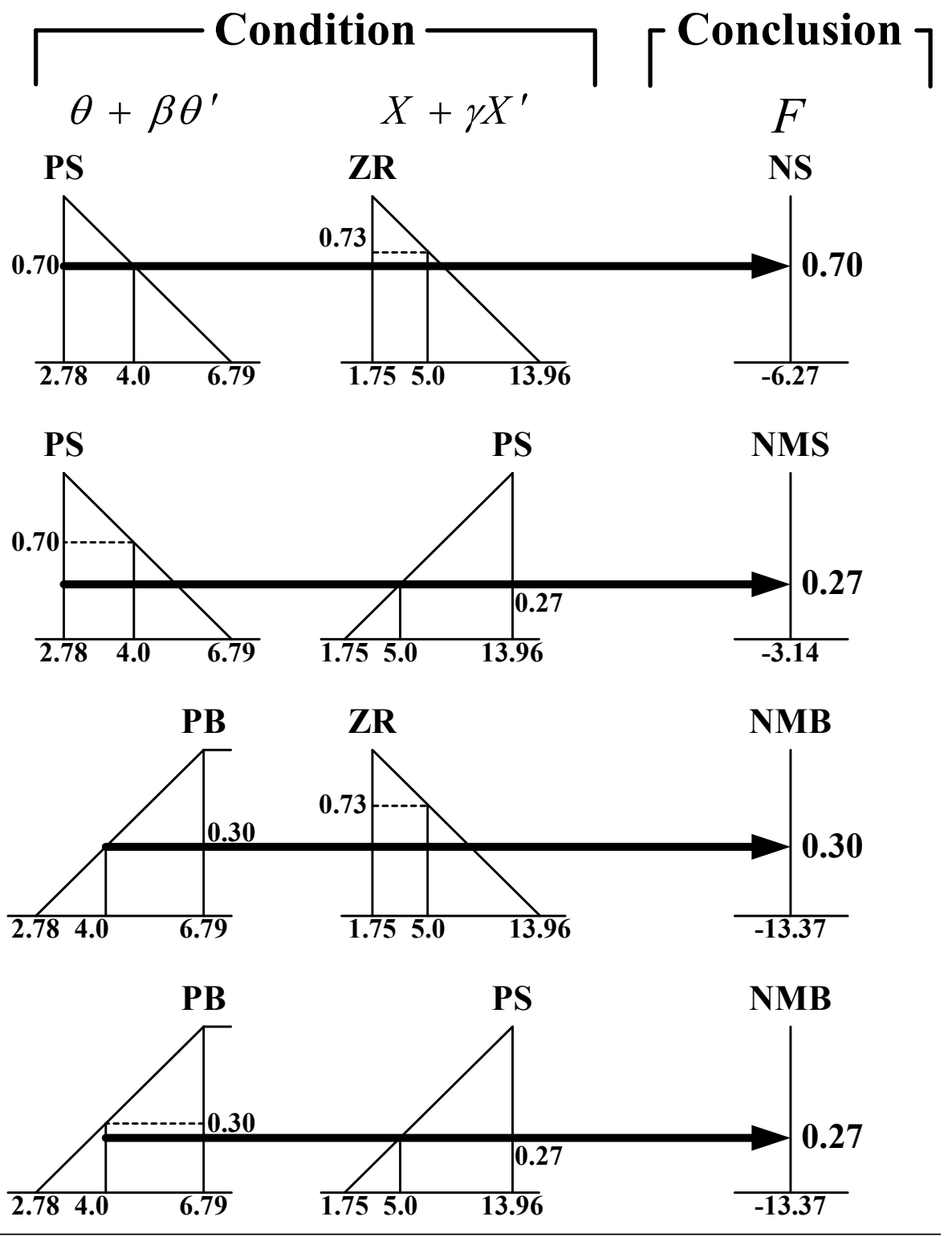

Fig. 12. Cutting by MIN value. 


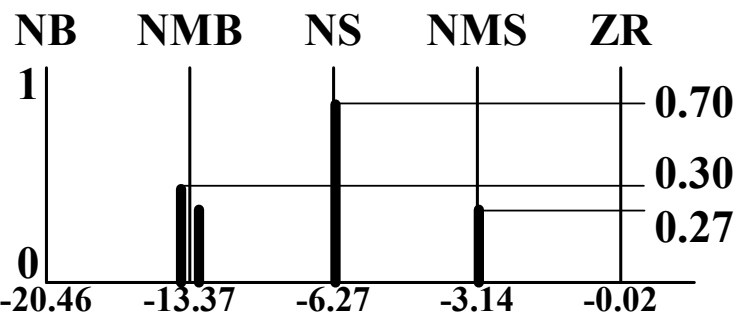

Fig. 13. Composition by MAX value.

We choose the pendulum angle $\theta_{\mathrm{t}}$, angular velocity $\theta^{\prime}{ }_{t}$, the cart displacement $X_{t}$ and its velocity $X^{\prime}{ }_{t}$ as input variables, and the force $F_{t}$ that moves the cart as output of the fuzzy controller, trying to identify the nonlinear characteristics of the human operator from the experimental time series data. Furthermore, we choose the combined variables $\theta_{t}+\beta \theta^{\prime}{ }_{t}$ and $X_{t}+\gamma X^{\prime}{ }_{t}$ as inputs so as to eliminate the complexity of the control rule table. The $\beta$ and $\gamma$ are the combination variables.

The values of $\beta$ and $\gamma$ are identified with the identification of membership functions and control rules by a trial and error method after repeating many simulations.

We can determine the output label and construct the operator's control rule for balancing the inverted pendulum.

\section{Chaos-entropy analysis and acquisition of individuality and proficiency using a fuzzy controller}

Figure 14 and Fig.15 show the membership functions of pendulum angle and its angular velocity, the membership function of cart displacement and its velocity, and the

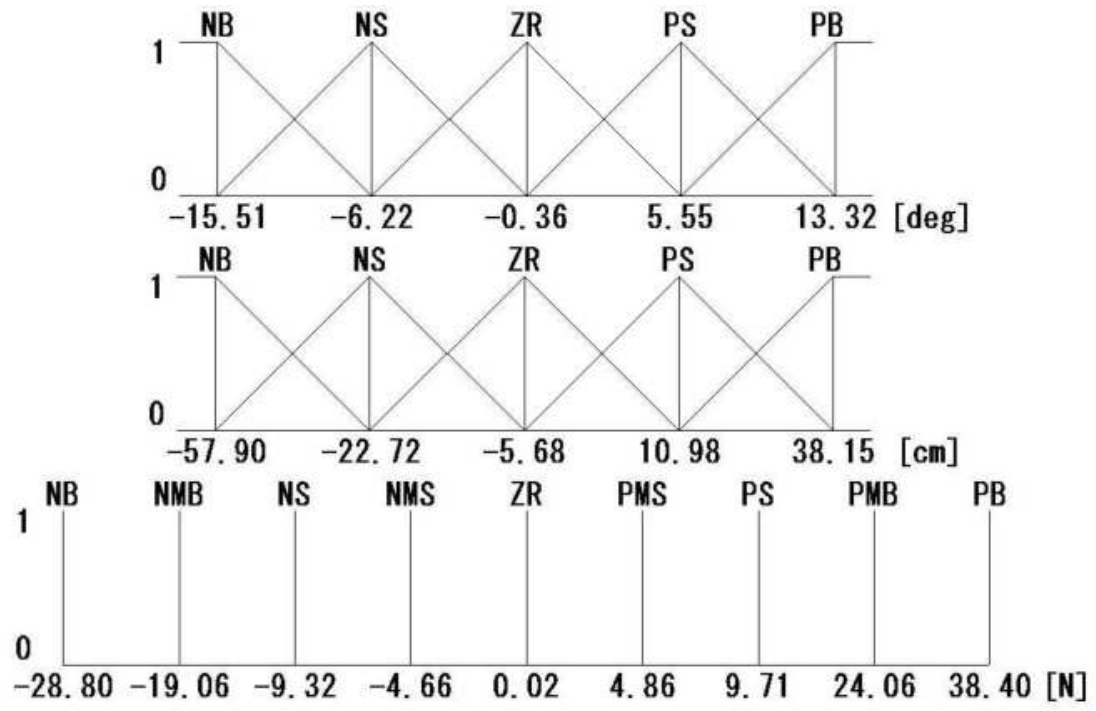

Fig. 14. Identified membership function (operator OT01) 

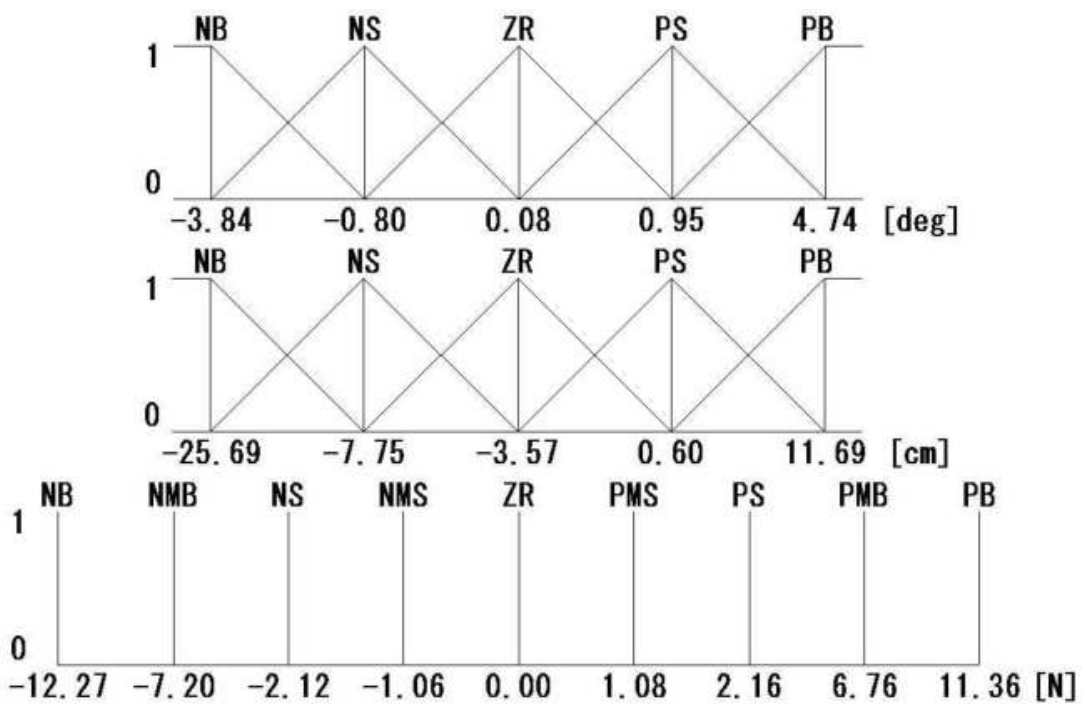

Fig. 15. Identified membership function (operator ME01)

\begin{tabular}{|c|c|c|c|c|c|c|}
\hline & \multicolumn{5}{|c|}{$\theta+\beta \theta^{\prime}$} \\
\hline & & NB & NS & ZR & PS & PB \\
\hline \multirow{5}{*}{$\begin{array}{l}x \\
x \\
\pm \\
x\end{array}$} & NB & PS & PMS & NMS & ZR & ZR \\
\hline & NS & PNB & PMS & NMS & NMB & ZR \\
\hline & ZR & PB & PS & ZR & NS & NMB \\
\hline & PS & ZR & PB & PMS & NMS & NMB \\
\hline & PB & ZR & ZR & PS & ZR & NS \\
\hline
\end{tabular}

(a) Human operator AT01 $\beta=0.0608, \gamma=0.2280$

\begin{tabular}{|c|c|c|c|c|c|c|}
\hline & \multicolumn{5}{|c|}{$\theta+\beta \theta^{\prime}$} \\
\hline & & NB & NS & ZR & PS & PB \\
\hline \multirow{5}{*}{$\begin{array}{l}\dot{x} \\
\dot{x} \\
\dot{x}\end{array}$} & NB & PMB & ZR & ZR & NMS & NB \\
\hline & NS & PMB & PMS & ZR & NS & NB \\
\hline & ZR & PMB & PMS & ZR & NMS & NS \\
\hline & PS & PB & PMS & ZR & NMS & NMB \\
\hline & PB & ZR & PS & PS & NMS & NS \\
\hline
\end{tabular}

(b) Human operator ME01 $\beta=0.0174, \gamma=0.0797$

\begin{tabular}{|c|c|c|c|c|c|c|}
\hline & \multicolumn{5}{|c|}{$\theta+\beta \theta^{\circ}$} \\
\hline & & NB & NS & ZR & PS & PB \\
\hline \multirow{5}{*}{$\begin{array}{l}\dot{x} \\
\dot{x} \\
\pm \\
\bar{x}\end{array}$} & NB & PMB & PMS & NMS & ZR & ZR \\
\hline & NS & PMB & PS & NMS & NB & ZR \\
\hline & ZR & PB & PMB & ZR & NMB & NB \\
\hline & PS & ZR & PB & PMS & NMS & NMB \\
\hline & PB & ZR & ZR & PMS & NMS & NMB \\
\hline
\end{tabular}

(c) Human operator OT01 $\beta=0.0451, \gamma=0.1619$

\begin{tabular}{|c|c|c|c|c|c|c|}
\hline & \multicolumn{5}{|c|}{$\theta+\beta \theta^{\prime}$} \\
\hline & & NB & NS & ZR & PS & PB \\
\hline \multirow{5}{*}{$\begin{array}{l}x \\
x \\
+ \\
x\end{array}$} & NB & PMB & PMS & NMS & NMB & NB \\
\hline & NS & PS & PMS & NMS & NMB & NB \\
\hline & ZR & PMB & PS & ZR & NS & NB \\
\hline & PS & PMB & PB & PMS & NMS & NS \\
\hline & PB & ZR & PB & PS & NMS & NMB \\
\hline
\end{tabular}

(d) Human operator ST01 $\beta=0.0595, \gamma=0.6806$

Fig. 16. Individual skill of each operator captured in fuzzy rules constructed from the experimental time series data

membership function (Singleton) for output force, which are identified from experimental time series data of Human Operator OT's 1st trial and ME's 1st trial. Figure 16 shows the individual skill of each operator captured in fuzzy rules constructed from the experimental time 
series data. It is seen that the fuzzy rules depend on the individual operator and are not symmetrical.

Entropy can be interpreted as a measure of the amount of disorder in the system, and the maximum entropy can be interpreted as a random process with a uniform probability.

Figure 17 shows the entropy ratio vs. number of trial for human operators NK and Ot. According to the results for the estimated entropy ratio, the simulated time series data have a large amount of disorder. The estimated entropy ratio of motion increases with the increase in proficiency.

Figure 18 shows the estimated dimension (degrees of freedom) of motion vs. the number of trials for operators NK and OT. The estimated number of degrees of freedom of motion increases with the increase in proficiency.

Figures 19-1 and 19-2 show examples of simulated waveforms with the identified fuzzy controller and measured waveforms that are similar in appearance for eight human operators.

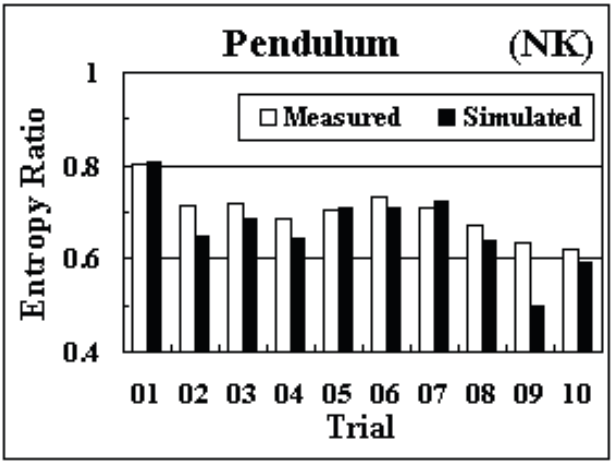

(a) Human operator NK

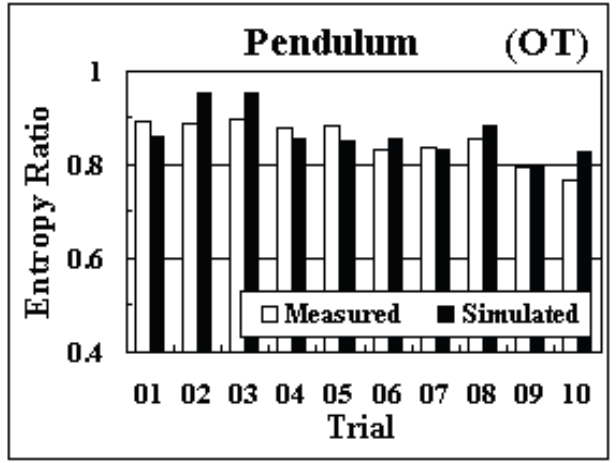

(b) Human operator OT

Fig. 17. Entropy ratio vs. number of trials by human operators (measured and simulated with the identified fuzzy controller).

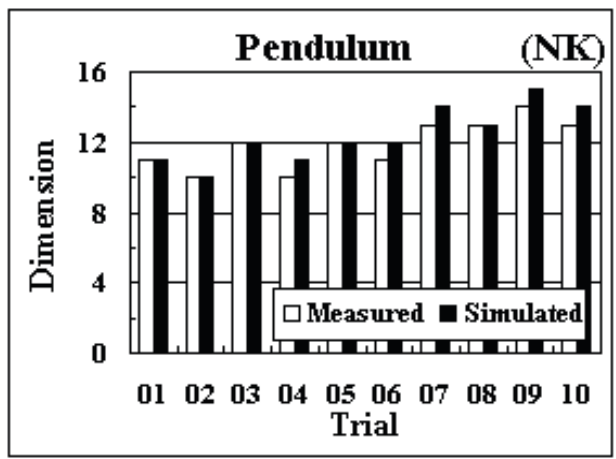

(a) Human operator NK

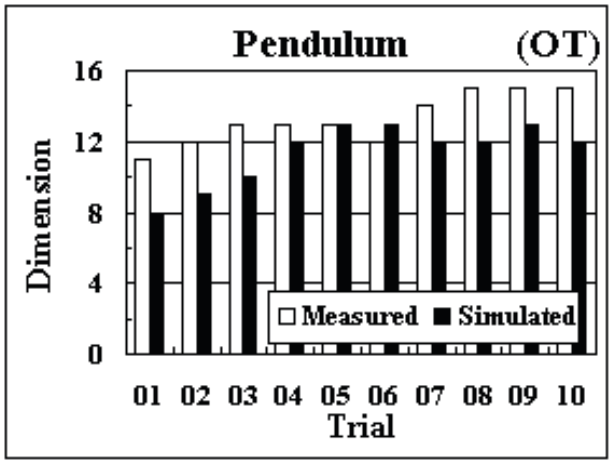

(b) Human operator OT

Fig. 18. Estimated dimension (degrees of freedom) of motion vs. number of trials by human operators (measured and simulated with the identified fuzzy controller). 


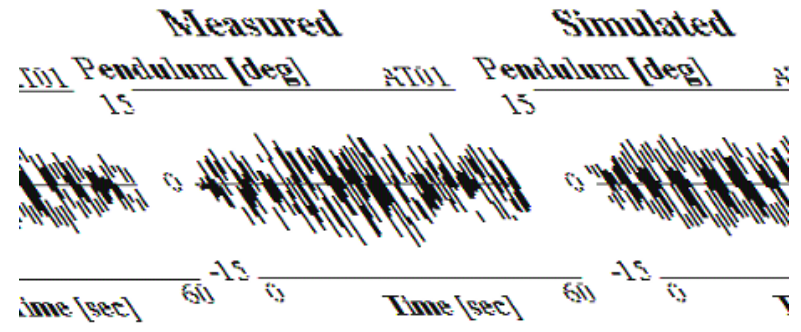

(a) Operator AT

Heasured

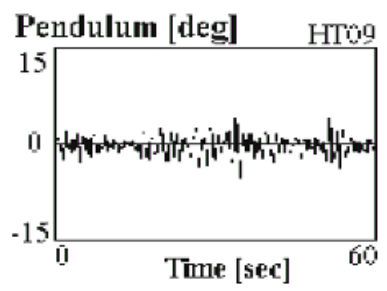

Simulated

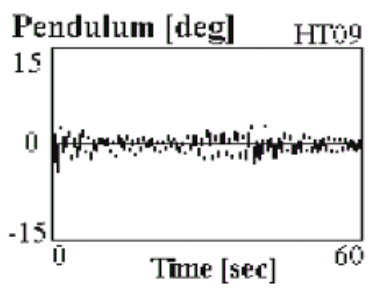

(b) Operator HT

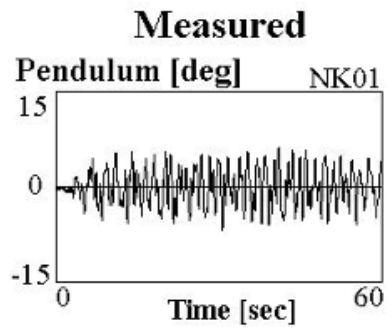

\section{Simulated}

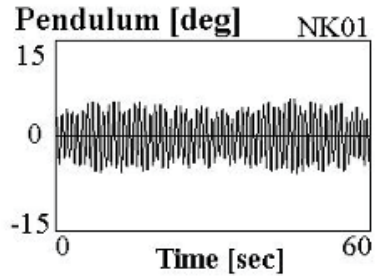

(c) Operator NK
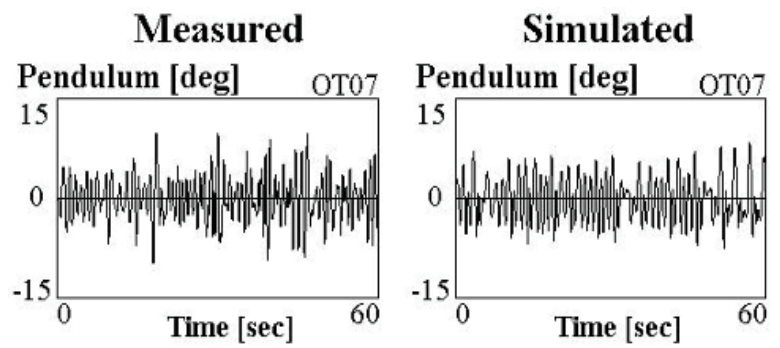

(d) Operator OT

Fig. 19-1. Examples of simulated waveforms with the identified fuzzy controller and measured waveforms that are similar in appearance for eight human operators. 
Measured

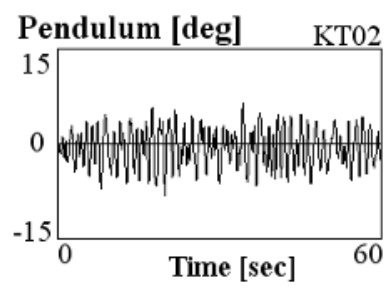

Simulated

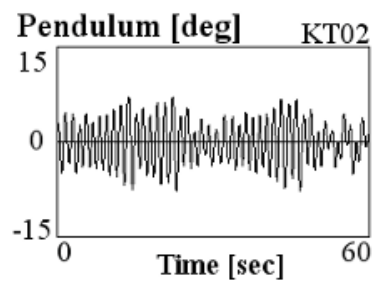

(e) Operator KT

Measured

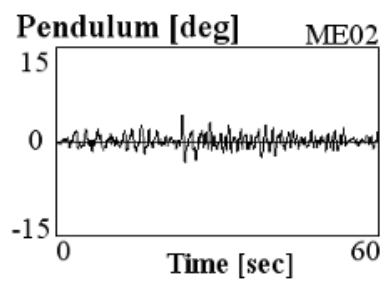

Simulated

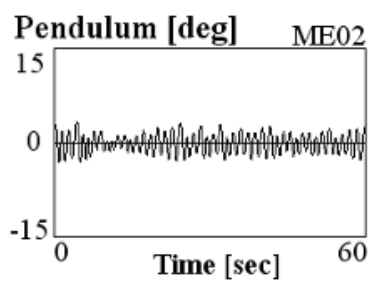

(f) Operator ME

Measured

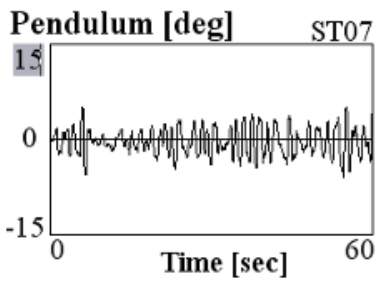

Simulated

Pendulum [deg] ST07

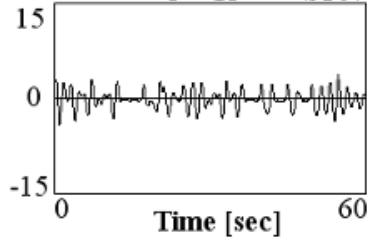

(g) Operator ST

Measured

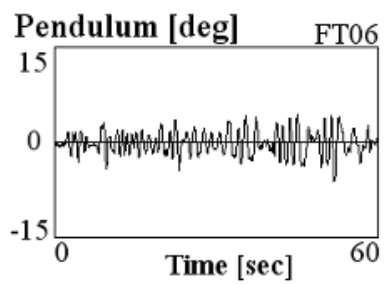

Simulated

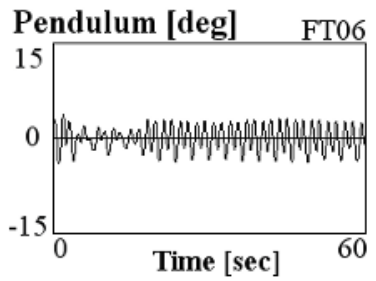

(h) Operator FT

Fig. 19-2. Examples of waveforms simulated with the identified fuzzy controller and measured waveforms that are similar in appearance for eight human operators. 
Figure 20 shows the entropy ratios for eight human operators and for simulations using an identified fuzzy controller to produce similar waveforms.

Figure 21 shows the estimated dimension (degrees of freedom) for eight human operators and for simulations using an identified fuzzy controller to produce similar waveforms.

The agreement between the simulated and experimental values for the number of degrees of freedom of motion and the entropy ratio is particularly good when the simulated waveform and the measured waveform are similar in appearance.

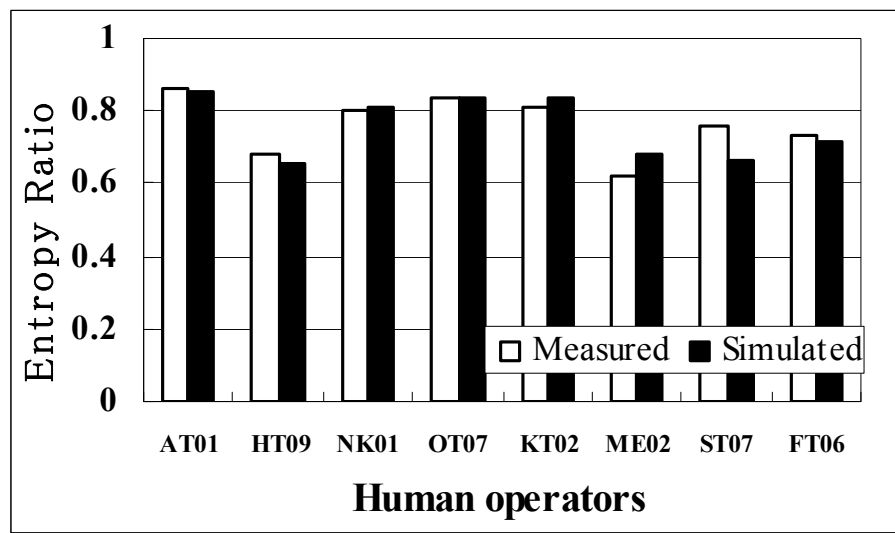

Fig. 20. Entropy ratios for eight human operators and for simulations using an identified fuzzy controller to produce similar waveforms.

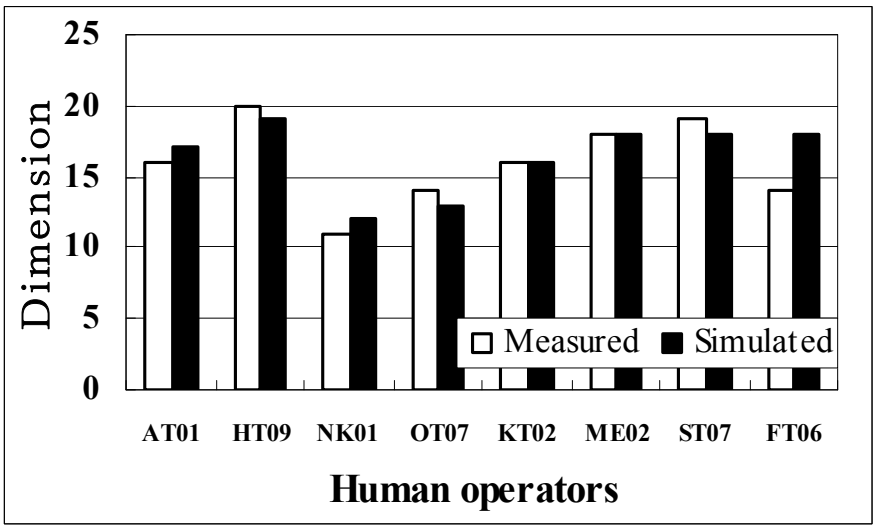

Fig. 21. Estimated dimension (degree of freedom) for eight human operators and for simulations using an identified fuzzy controller to produce similar waveforms.

\section{Conclusion}

we have demonstrated that the fuzzy controller identified from the measured time series data for each trial for each human operator clearly exhibited the human-generated decisionmaking characteristics, exhibiting chaos and a large amount of disorder. We have also 
shown that the estimated number of degrees of freedom of motion increases and the estimated amount of disorder decreases with the increase in proficiency in the fuzzy control simulation. In addition, we have shown that the agreement between the experimental results and the fuzzy simulation for the number of degrees of freedom and for the entropy ratio of motion is particularly good when the measured waveform and the simulated waveform are similar in appearance. Accordingly, it was clarified that a simple fuzzy controller can be very useful for identifying the individuality and proficiency of a human operator when stabilizing an unstable system.

At present, despite notable differences in the behaviors of machinery and human beings, most research on man-machine systems has dealt with the linear characteristics of human behavior. There appear to be few studies and a number of unknowns regarding both the nonlinear characteristics of human behavior in an inherently unstable man-machine system and the learning process of human operators for objects that are difficult to control (Kawazoe et al., 2008, 2009) .

The excessive number of degrees of freedom appears to provide considerable advantages. In several cases, a more flexible instrument, which is certainly much more challenging to work with, has undeniable advantages that provide better results. An experienced master will always prefer an instrument with a greater number of degrees of freedom over an instrument that is easier to use but constrains the worker. For example, a bicycle is harder to control than a tricycle, but anyone who has mastered a bicycle will probably never want to ride a tricycle again. The bicycle is preferred because in the hands of an experienced rider, it is more flexible and maneuverable and becomes more stable than the tricycle. Similarly, lightweight children's skates with their wide blades are less flexible and maneuverable than narrow-bladed speed skates. The practical problem of acquiring dexterity occurs in the early stages of skill development. This fascinating and extremely important area can move us closer to the deepest, concealed caches of knowledge about the human brain and its function (Bernstein, 1996). Real intelligence in autonomous robots appears to be expressed by dexterity in humans or other living creatures as complex systems, and research and development are required to realize intellectual autonomous robots.

Confusion and doubt arise among individuals who are involved in robotics research and development for a long time because numerous robot control theories have been proposed.

Dexterous dynamic actions required for humanoid biped robots, for example, are difficult to achieve through the current standard control strategy for humanoid robots based on the asymptotic convergence to the successive desired states with small fluctuations. Thus, a new and alternative approach is necessary. In the future, we would like to apply the simple nonlinear optimal control of various movements to make full use of instability as a source of driving force.

\section{References}

Baierlein, R., Atoms and Information Theory, (1971), W. H. Freeman and Co., San Francisco, Chapter 3.

Baker, G. L. and Gollub, J. P., Chaotic dynamics: an introduction, Cambridge University Press, (1996), pp. 86-87.

Bernstein, N. A., Dexterity and its Development, Latash, M. and Turvey, M. (eds.), LEA Publishers, New Jersey, (1996).

Gesell, A., 1945, The embryology of behavior, New York: Harper. 
Kawazoe, Y., Manual control and computer control of an inverted pendulum on a cart, Proc. of the First International Conf. on Motion and Vibration Control, (1992), pp.930 -935.

Kawazoe, Y. and Ju, D. Y., Nonlinear characteristics of human operator with the stabilizing control of an inverted pendulum on a cart, Proc. 2nd Int. Conf. on Motion and Vibration Control, (1994), pp.645- 650.

Kawazoe, Y., Nonlinear characteristics of a human operator during stabilizing control of an inverted pendulum on a cart: Fuzzy identification from experimental time series data and Fuzzy control simulation, Motion and vibration control in Mechatronics, Edited by Seto, K., Mizuno, T. and Watanabe, T., (1999), pp.133-138.

Kawazoe Y., Measurement and Analysis of Chaotic Behavior of Human Operator Stabilizing an Inverted Pendulum on a Cart, Proc. ICMA2000-Human Friendly Mechatronics, pp.457-462 (2000).

Kawazoe, Y., Fuzzy Identification of Chaotic and Complex Behavior of Human Operator Stabilizing an Inverted Pendulum on a Cart, Proc. 6th Int. Symposium on Artificial Life E Robotics, pp.9-12. (2001a)

Kawazoe, Y., Measurement of Chaotic Behavior of Human Operator stabilizing an Inverted Pendulum and Its Fuzzy Identification from Time Series Data, J. Robotics $\mathcal{E}$ Mechatronics, 13-1, pp.23- 29.(2001b)

Kawazoe, Y., Ikura, Y., Uchiyama, K., and Kaise, T., Chaos-Entropy Analysis and Acquisition of Individuality and Proficiency of Human Operator's Skill Using a Neural Controller, Journal of System Design and Dynamics, Vol. 2, No. 6, (2008), pp. 1351-1363.

Kawazoe, Y., Ikura, Y., Kaise, T., and Matsumoto, J., Chaos-Entropy Analysis and Acquisition of Human Operator's Skill Using a Fuzzy Controller: Identification of Individuality During Stabilizing Control of an Inverted Pendulum, Journal of System Design and Dynamics, Vol. 3, No. 6, (2009), pp. 932-943.

Mane, R. On the dimension of the compact invariant sets of certain nonlinear maps, in Rand, D. A. and Young L. S. (eds.), ibid, Vol. 898, (1981), Springer Verlag, Berlin, pp. 230242.

Newell, K.M.,van Emmerik,R.E.A.,1989,The acquisition of coordination: preliminary analysis of learning to write, Hum Mov Sci., 8, 17-32.

Taga, G., Dynamic Design of Brain and Body, (2002), Kaneko Shobo. (in Japanese)

Takens, F., Detecting strange attracters in turbulence, in Rand, D. A. and Young, L. S. (eds.), Lecture Notes in Mathematics, Vol. 898, pp. 366-381, (1981), Springer-Verlag, Berlin.

Wolf, A., Swift, J. B., Swinney H. L., and Vastano J. A., Determining Lyapunov Exponents From a Time Series, Physica, 16D, (1985). pp. 285-317. 


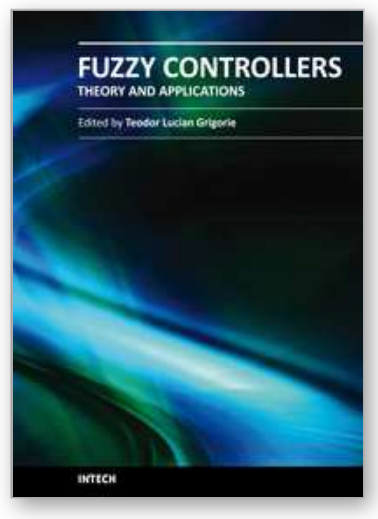

\author{
Fuzzy Controllers, Theory and Applications \\ Edited by Dr. Lucian Grigorie
}

ISBN 978-953-307-543-3

Hard cover, 368 pages

Publisher InTech

Published online 28, February, 2011

Published in print edition February, 2011

Trying to meet the requirements in the field, present book treats different fuzzy control architectures both in terms of the theoretical design and in terms of comparative validation studies in various applications, numerically simulated or experimentally developed. Through the subject matter and through the inter and multidisciplinary content, this book is addressed mainly to the researchers, doctoral students and students interested in developing new applications of intelligent control, but also to the people who want to become familiar with the control concepts based on fuzzy techniques. Bibliographic resources used to perform the work includes books and articles of present interest in the field, published in prestigious journals and publishing houses, and websites dedicated to various applications of fuzzy control. Its structure and the presented studies include the book in the category of those who make a direct connection between theoretical developments and practical applications, thereby constituting a real support for the specialists in artificial intelligence, modelling and control fields.

\title{
How to reference
}

In order to correctly reference this scholarly work, feel free to copy and paste the following:

Yoshihiko Kawazoe (2011). Acquisition and Chaos-Entropy Analysis of Individuality and Proficiency of Human Operator's Skill Using a Fuzzy Controller, Fuzzy Controllers, Theory and Applications, Dr. Lucian Grigorie (Ed.), ISBN: 978-953-307-543-3, InTech, Available from: http://www.intechopen.com/books/fuzzy-controllerstheory-and-applications/acquisition-and-chaos-entropy-analysis-of-individuality-and-proficiency-of-humanoperator-s-skill-us

\section{INTECH}

open science | open minds

\section{InTech Europe}

University Campus STeP Ri

Slavka Krautzeka 83/A

51000 Rijeka, Croatia

Phone: +385 (51) 770447

Fax: +385 (51) 686166

www.intechopen.com

\section{InTech China}

Unit 405, Office Block, Hotel Equatorial Shanghai

No.65, Yan An Road (West), Shanghai, 200040, China 中国上海市延安西路65号上海国际贵都大饭店办公楼 405 单元

Phone: $+86-21-62489820$

Fax: +86-21-62489821 
(C) 2011 The Author(s). Licensee IntechOpen. This chapter is distributed under the terms of the Creative Commons Attribution-NonCommercialShareAlike-3.0 License, which permits use, distribution and reproduction for non-commercial purposes, provided the original is properly cited and derivative works building on this content are distributed under the same license. 